\title{
Study of Active Screening to Detect Un-Registered Open Cases of Tuberculosis in Rural Areas of Capital District of Rajasthan, India
}

\author{
Praveen Kumar Anand1 ${ }^{*}$, Mradula Singh², Chet Ram Meena', Swarn Lata ${ }^{3}$, Anil Jangid ${ }^{2}$, \\ Megh Raj Devanda ${ }^{2}$, Murli Lal Mathur', Monika Rathore ${ }^{4}$, Bhanwar Manohar Singh', \\ Pankaj Kumar ${ }^{1}$
}

${ }^{1}$ ICMR - National Institute for Implementation Research on Non Communicable Diseases, New Pali Road, Jodhpur, Rajasthan, India

${ }^{2}$ Model Rural Health Research Unit, Department of Health Research, Government of India, Bhanpur Kalan, Jaipur, Rajasthan, India ${ }^{3}$ Department of Pathology, Dr. Sampoornanand Medical College, Jodhpur, Rajasthan, India

${ }^{4}$ Department of Preventive \& Social Medicine, Sawai Man Singh Medical College \& Associated Hospitals, Jaipur, Rajasthan, India Email: *ananddmrc@yahoo.co.in

How to cite this paper: Anand, P.K., Singh, M., Meena, C.R., Lata, S., Jangid, A., Devanda, M.R., Mathur, M.L., Rathore, M., Singh, B.M. and Kumar, P. (2021) Study of Active Screening to Detect Un-Registered Open Cases of Tuberculosis in Rural Areas of Capital District of Rajasthan, India. Journal of Tuberculosis Research, 9, 276-283. https://doi.org/10.4236/jtr.2021.94026

Received: October 13, 2021

Accepted: December 20, 2021

Published: December 23, 2021

Copyright $\odot 2021$ by author(s) and Scientific Research Publishing Inc. This work is licensed under the Creative Commons Attribution International License (CC BY 4.0).

http://creativecommons.org/licenses/by/4.0/ (c) (i) Open Access

\begin{abstract}
Tuberculosis is one among top 10 causes of deaths worldwide. Access to effective diagnosis by every TB patient is one among five missions of Global Plan. Active case finding among vulnerable groups has been the key focus area of National Strategic Plan to control TB in India 2017-25. This crosssectional study was carried out to find out the additional open cases of TB through active screening of general \& rural population in selected villages of Jaipur, the capital district in Rajasthan state of India. An active screening team of trained staff visited each house-hold in selected study villages for interviewing the head of house-hold or other adult member available at the time of visit. Family members of age $\geq 15$ years were screened for complain of chronic cough of duration 2 weeks or more with study questionnaire. Presumptive cases found at screening were referred for seeking medical consultations from nearest designated microscopy centre. The referred patients were followed up on telephone up to five times for results of medical consultancies. Laboratory technicians of catchment DMCs were also followed up for verification of results. Total 19,306 individuals were covered. Study included 52.8\% male, $69.3 \% \geq 15$ years age, and $99.3 \%$ Hindu participants. There were total 153 presumptive TB patients. Out of these, total 10 cases were confirmed as TB. The yield of detection was 0.51 cases $/ 1000$ population screened. Study finds active screening as a feasible and effective strategy in detecting additional cases of TB from general and rural population.
\end{abstract}




\section{Keywords}

Tuberculosis, Active Screening, Rural, Adult, Community

\section{Introduction}

Tuberculosis (TB) is one of the top 10 causes of deaths worldwide. Eight countries of world, including India, account for the two thirds of global total of TB cases [1]. The Global Plan and Stop TB Partnership 2006-2015 visualizes a TB free world. To ensure the TB free world, access to effective diagnosis, treatment and cure by every TB patient is one among five missions of Global Plan [2]. India is still a highest TB burden country globally in regards of absolute numbers of incident cases each year. Mortality due to this disease is the third leading cause of years of life lost (YLLs), in India [3]. Active case finding (ACF) among vulnerable groups is key focus area in NSP 2017-25. Difficult to reach villages is one priority area in rural population for ACF [3].

Many studies carried out in different countries have shown that active screening of population yields detection of large number of cases of TB which were not detected by passive screening [4] [5] [6] [7] [8].

RNTCP has already achieved the WHO recommended target of new sputum positive case detection rate of more than $70 \%$ and the treatment success rate of $>85 \%$ in 2007 and persistently maintaining these since then [9].

Active case finding strategy for TB detection is focus of national strategic plan for TB elimination 2017-2025 in India. It has prioritized certain areas for active case finding not only from urban and rural areas but also from tribal areas [3].

In order to achieve more than $90 \%$ detection, active screening of general adult population may be desirable. For effective control of tuberculosis, attempt should be made to identify and treat all open cases that can transmit the disease in general population. This can in long run help break the transmission cycle of the disease. So far, active case search for cases of TB has been recommended in the certain prioritized vulnerable population groups. This study was carried out to find out the additional open cases of TB through active screening of chronic coughers (of 2 weeks or more duration) in general rural population in selected villages of Jaipur, the capital district in Rajasthan state of India.

\section{Materials \& Methods}

Study design: Cross-sectional community based study

Study setting: This study was carried out at Model Rural Health Research Unit, Jaipur, a unit of Department of Health Research, Government of India [10]. Jaipur is the capital district of state of Rajasthan.

Sample size: Assuming prevalence of 3 smear positive cases/1000 population [11] and 70\% case detection rate by TB programme, it was expected that approximately 1 case/1000 population remains undetected. It was decided to carry 
out active screening of population to detect at least 20 un-registered TB cases. It was therefore decided to screen 20,000 individuals.

Sampling design: Two stage simple random sampling design was adopted. In first stage, 2 tehsils namely Jamwa Ramgarh and Sanganer were selected from the 17 tehsils of district Jaipur [12]. In second stage from each of the selected tehsil, 5 villages were randomly selected. In total we selected 10 villages from 2 tehsils of district Jaipur.

Active screening team: An active screening team was composed involving scientist, project scientist and technical staff posted at Model Rural Health Research Unit. All team members were formally sensitized about the project protocol, its objectives, \& methodologies, and trained in seeking consent from participants, administration of study questionnaire, and collection of data and referral of suspected TB cases to adjoining Designated Microscopic Centre, and telephonic follow ups. Scientist's role was to brief the study participant about the purpose, procedure of study and receiving the consent. Project scientist's role was to administer the study questionnaire and interviewing the participant. Technical staff was assigned the role of getting signature \& managing consent forms along with study questionnaire. Project scientist also followed the presumptive TB cases telephonically for follow up actions after active screening. Laboratory technicians posted at catchment DMCs of the selected study villages were also sensitized \& trained about the purpose of study and its protocol.

Active case-finding strategy: Each house-hold in selected study villages were visited by members of the active screening team for interviewing the head of house-hold or other adult member available at the time of visit. Adult family members of age 15 years or more were screened for complain of chronic cough of duration 2 weeks or more with study questionnaire. A referral slip was handed over to the chest symptomatic and motivated him/her for seeking medical consultations from nearest designated microscopy centre. The referred chest symptomatic patients were followed up on telephone for results of medical consultancies. In case of unavailability of telephone, telephone of the person in neighborhood was used in following up the referred patient. Laboratory technicians of catchment DMCs were also followed up for verification of results of medical consultancies as reported by referred patients. In case, chest symptomatic failed to report at microscopic centre, such cases were again contacted telephonically and motivated to report to microscopic centre for diagnosis. Study was carried out during 2014-2017.

Operational case definition of "Presumptive TB case": In the study, any person of age 15 years or more with complain of cough for last two weeks period or more was defined as a "Presumptive TB case". The case ascertainment was irrespective of the gender of participant and sputum production.

\section{Results}

Details of the population covered are given in Table 1 . There were a total of 19,544 individuals residing in these total 10 study villages. Out of these 19,544 
village residents, 19,306 individuals were covered in the study. Total 238 individuals could not contact because of either lock in the door or migrated out. Out of the covered 19,306 study participants, 12,803 (66.3\%) individuals were covered from 5 villages of block Jamwa Ramgarh and 6503 (33.7\%) individuals were covered from 5 villages of block Sanganer of district Jaipur. Study included $52.8 \%$ male, $69.3 \% \geq 15$ years age, and $99.3 \%$ Hindu participants.

Table 2 elaborates on No. of persons found with known TB status, presumptive TB cases i.e. persons with history of cough for 2 weeks duration, and No. of

Table 1. General characteristics of the studied population $(\mathrm{N}=19,306)$.

\begin{tabular}{ccccc}
\hline \multirow{7}{*}{ Characteristic } & $\begin{array}{c}\text { Block Jamwa } \\
\text { Ramgarh } \\
(\mathrm{n}=12,803)\end{array}$ & $\begin{array}{c}\text { Block } \\
\text { Sanganer } \\
(\mathrm{n}=6503)\end{array}$ & $\begin{array}{c}\text { Total } \\
(\mathrm{n}=19,306)\end{array}$ \\
\hline \multirow{2}{*}{ Gender } & Male & $6889(53.8 \%)$ & $3308(50.86 \%)$ & $10,197(52.8 \%)$ \\
& Female & $5895(46.0 \%)$ & $3178(48.86 \%)$ & $9073(46.9 \%)$ \\
Age group & Information missing & $19(0.14 \%)$ & $17(0.26 \%)$ & $36(0.2 \%)$ \\
\cline { 2 - 5 } & Information missing & $75(0.58 \%)$ & $92(1.41 \%)$ & $167(0.9 \%)$ \\
\hline \multirow{2}{*}{ Religion } & Hindu & $12,803(100 \%)$ & $6371(97.9 \%)$ & $19,174(99.3 \%)$ \\
& Muslim & $0(0 \%)$ & $132(2.02 \%)$ & $132(0.7 \%)$ \\
\hline
\end{tabular}

Table 2. Details of known TB cases, presumptive TB cases referred and NEW detected TB cases.

\begin{tabular}{ccccc}
\hline Characteristic & $\begin{array}{c}\text { Total } \\
(\mathrm{n}=19,306)\end{array}$ & $\begin{array}{c}\text { Block Jamwa } \\
\text { Ramgarh } \\
(\mathrm{n}=12,803)\end{array}$ & $\begin{array}{c}\text { Block } \\
\text { Sanganer } \\
(\mathrm{n}=6503)\end{array}$ & $\begin{array}{c}\mathrm{p} \text { value } \\
\text { of } \chi^{2} \text { test }\end{array}$ \\
\hline $\begin{array}{c}\text { Known TB cases found } \\
\text { Old TB cases who received } \\
\text { ATT }>\text { 2 years before }\end{array}$ & 59 & $27(0.2 \%)$ & $32(0.5 \%)$ & 0.0008 \\
$\begin{array}{c}\text { OLD TB cases who } \\
\text { received ATT within }\end{array}$ & 12 & $4(0.03 \%)$ & $8(0.12 \%)$ & 0.01 \\
$\begin{array}{c}\text { (6 months to } \leq 2 \text { years } \\
\text { New TB cases receiving }\end{array}$ & 17 & $11(0.08 \%)$ & $6(0.09 \%)$ & 0.88 \\
$\begin{array}{c}\text { ATT within last 6 months } \\
\text { Information missing }\end{array}$ & 13 & $7(0.05 \%)$ & $6(0.09 \%)$ & 0.34 \\
$\begin{array}{c}\text { Presumptive TB cases found } \\
\text { NEW TB case detected }\end{array}$ & 153 & $111(0.86 \%)$ & $42(0.64 \%)$ & 0.10 \\
Yield of & 10 & $8(0.06 \%)$ & $2(0.03 \%)$ & 0.35 \\
cases/1000 population & 0.51 & 0.62 & 0.30 & $0.35^{*}$ \\
\hline
\end{tabular}

Note: $\mathrm{p}$ value estimated by comparing 2 person-time rates in Open-Epi software. 
cases of TB detected out of these presumptive TB cases after their referral to public health system. There were total 59 persons who reported to suffered with TB in their life time. Out of these 59 known cases of TB, 27 and 32 cases were from block Jamwa Ramgarh and Block Sanganer respectively. The difference between proportions of known TB cases in persons from block Jamwa Ramgarh and block Sanganer was statistically significant $(\mathrm{p}<0.0008)$. Similarly, the difference between proportions of known TB cases who received anti-tubercular therapy $>2$ years ago among persons from block Jamwa Ramgarh and block Sanganer was statistically significant $(\mathrm{p}<0.01)$. Proportion of old TB cases who received anti-tubercular therapy 6 months $-<2$ years ago also significantly differed between persons in these 2 blocks $(\mathrm{p}<0.001)$.

Both blocks were statistically similar in respect to the difference in proportion of New TB cases on ATT within last 6 months. There were total 153 persons who reported to suffer with chronic cough in study on active screening. However, the difference in proportion of presumptive TB cases was statistically insignificant. Out of the 153 presumptive TB cases, total 10 cases were confirmed as TB. The yield of detecting TB cases by active screening was 0.51 cases $/ 1000$ population screened. Both blocks were similar statistically in this regards.

\section{Discussions}

A mass tuberculosis (TB) community survey in Cambodia resulted in detection of additional new TB cases which was found associated with rapid reduction in subsequent TB incidence [4].

An active house-to-house survey, using chronic cough (2 weeks or more) enquiry as a screening tool, identified $18 \%$ undiagnosed smear-positive cases among chronic coughers. Findings suggested that active case finding could supplement DOTS to yield additional smear-positive TB cases [5].

An active-case finding strategy evaluation study in rural population of Ethiopia demonstrated 2.5 undetected TB cases in community for every smear positive $\mathrm{TB}$ case receiving treatment during the study period [6].

A cluster randomized trial in Zimbabwe compared two active case-finding strategies for community based diagnosis of symptomatic smear-positive tuberculosis. Six rounds of active case-findings were undertaken either by mobile van or by door-to-door visits. Active case-finding intervention in area resulted in decline in overall prevalence of culture-positive tuberculosis from 6.5 per 1000 adults to 3.7 per 1000 adults [7].

"Cough Officer Screening" an active screening system in Taiwan improved detection of TB by reducing the delay from infection to diagnosis [8].

Markos A et al. opined that control programmes should move from simple passive, to a more systematic active case finding in order to accelerate TB control [13].

Arshad et al. in their systematic review and meta-analysis determined the yield (defined as the ratio between number of cases detected and individuals screened) of active case finding or active screening programmes of tuberculosis among 
new immigrants moving from areas of high tuberculosis incidence to areas of low incidence at the point of entry. The proportion of screened immigrants with active pulmonary tuberculosis ranged between 10 to 100 times greater than the prevalence measured in the general population of the host country [14].

Ochoa et al. compared the yield of active tuberculosis case detection among risk groups during home visits with passive detection among patients at health services. Authors demonstrated that active case finding in groups at risk during home visits increases the case detection rate in the population and permits the identification of cases that may not be detected through passive case finding at health facility level. The overall case detection rate of TB increased from 6.7/100,000 during passive detection at health services before the intervention to $26.2 / 100,000$ inhabitants when passive detection was complimented by active case finding [15].

Another study to evaluate the impact that Family Health Programme (FHP) team training and active surveillance have on the detection of TB cases in Brazil, found the number of TB cases detected increased significantly [16].

The impact of current interventions to improve early detection of tuberculosis (TB) seems to have been saturated. There is growing evidence of a large pool of undetected TB in the community. Could mass, community-wide screening be of benefit in some situations? If so, what screening tools and approaches should be used? WHO is in the process of seeking answers to these questions and developing guidelines on systematic screening for active TB [17]. Indian studies have also found out the additional case detection with active case finding in selected population groups [18] [19] [20] [21].

This study has demonstrated the occurrence of hidden cases of tuberculosis in general rural community. If not detected early, they may continue to infect other susceptible individuals and propagating the TB transmission in community. National strategic plan for tuberculosis of India 2017-2025 recommends for active case findings in select priority groups, including hard to reach villages in population [3]. This study supplements the recommendation of national strategic plan for TB. The study also suggests for implementing active screening in general rural population.

\section{Conflicts of Interest}

The authors declare no conflicts of interest regarding the publication of this paper.

\section{Source of Funding}

Study was funded by Model Rural Health Research Unit, Bhanpur Kalan, Jaipur. Unit supported the travel expenditures and provided the technical staff for active screening \& data collection.

\section{References}

[1] World Health Organization (2020) Global Tuberculosis Report 2020. https://www.who.int/publications-detail-redirect/9789240013131 
[2] Maher, D. (2006) The Global Plan to Stop TB 2006-2015: Actions for Life: Towards a World Free of Tuberculosis. The International Journal of Tuberculosis and Lung Disease, 10, 240-241.

[3] Government of India (2017) Revised National Tuberculosis Control Programme. National Strategic Plan for Tuberculosis Elimination 2017-2025.

[4] Okada, K., Onozaki, I., Yamada, N., Yoshiyama, T., Miura, T., Saint, S., et al. (2012) Epidemiological Impact of Mass Tuberculosis Screening: A 2-Year Follow-Up after a National Prevalence Survey. The International Journal of Tuberculosis and Lung Disease, 16, 1619-1624. https://doi.org/10.5588/ijtld.12.0201

[5] Sekandi, J.N., Neuhauser, D., Smyth, K. and Whalen, C.C. (2009) Active Case Finding of Undetected Tuberculosis among Chronic Coughers in a Slum Setting in Kampala, Uganda. The International Journal of Tuberculosis and Lung Disease, 13, 508 513.

[6] Yimer, S., Holm-Hansen, C., Yimaldu, T. and Bjune, G. (2009) Evaluating an Active Case-Finding Strategy to Identify Smear-Positive Tuberculosis in Rural Ethiopia. The International Journal of Tuberculosis and Lung Disease, 13, 1399-1404.

[7] Corbett, E.L., Bandason, T., Duong, T., Dauya, E., Makamure, B., Churchyard, G.J., et al. (2010) Comparison of Two Active Case-Finding Strategies for CommunityBased Diagnosis of Symptomatic Smear-Positive Tuberculosis and Control of Infectious Tuberculosis in Harare, Zimbabwe (DETECTB): A Cluster-Randomised Trial. The Lancet, 376, 1244-1253. https://doi.org/10.1016/S0140-6736(10)61425-0

[8] Lin, C.H., Tsai, C.H., Liu, C.E., Huang, M.L., Chang, S.C., Wen, J.H. and Chai, W.H. (2010) "Cough Officer Screening" Improves Detection of Pulmonary Tuberculosis in Hospital In-Patients. BMC Public Health, 10, Article No. 238.

https://doi.org/10.1186/1471-2458-10-238

https://bmcpublichealth.biomedcentral.com/articles/10.1186/1471-2458-10-238

[9] Government of India (2012) Revised National Tuberculosis Control Programme. Annual Status Report. TB India 2012.

[10] Government of India (2018) Annual Report 2017-18. Department of Health Research, Ministry of Health \& Family Welfare.

[11] Bhat, J., Rao, V.G., Gopi, P.G., Yadav, R., Selvakumar, N., Tiwari, B., et al. (2009) Prevalence of Pulmonary Tuberculosis amongst the Tribal Population of Madhya Pradesh, Central India. International Journal of Epidemiology, 38, 1026-1032. https://doi.org/10.1093/ije/dyp222

[12] Jaipur-Rajasthan Administration. Jaipur Rajasthan Official Website.

[13] Abebe, M., Doherty, M., Wassie, L., Demissie, A., Mihret, A., Engers, H., et al. (2012) TB Case Detection: Can We Remain Passive While the Process Is Active? The Pan African Medical Journal, 11, Article No. 50.

[14] Arshad, S., Bavan, L., Gajari, K., Paget, S.N.J. and Baussano, I. (2010) Active Screening at Entry for Tuberculosis among New Immigrants: A Systematic Review and Meta-Analysis. European Respiratory Journal, 35, 1336-1345. https://doi.org/10.1183/09031936.00054709

[15] González-Ochoa, E., Brooks, J.L., Matthys, F., Calisté, P., Armas, L. and Van der Stuyft, P. (2009) Pulmonary Tuberculosis Case Detection through Fortuitous Cough Screening during Home Visits. Tropical Medicine \& International Health, 14, 131 135. https://doi.org/10.1111/j.1365-3156.2008.02201.x

[16] Façanha, M.C., Melo, M.A., de Fátima Vasconcelos, F., de Sousa, J.R.P., de Sousa Pinheiro, A., Porto, I.A., et al. (2009) Health Team Training and Active Community Surveillance: Strategies for the Detection of TB Cases. Jornal Brasileiro de Pneumo- 
logia, 35, 449-454. https://doi.org/10.1590/S1806-37132009000500010

[17] Lönnroth, K., Corbett, E., Golub, J., Godfrey-Faussett, P., Uplekar, M., Weil, D., et al. (2013) Systematic Screening for Active Tuberculosis: Rationale, Definitions and Key Considerations. The International Journal of Tuberculosis and Lung Disease, 17, 289-298. https://doi.org/10.5588/ijtld.12.0797

[18] Shewade, H.D., Gupta, V., Satyanarayana, S., Kharate, A., Sahai, K.N., Murali, L., et al. (2018) Active Case Finding among Marginalised and Vulnerable Populations Reduces Catastrophic Costs Due to Tuberculosis Diagnosis. Global Health Action, 11, Article ID: 1494897. https://doi.org/10.1080/16549716.2018.1494897

[19] Chadha, V.K. and Praseeja, P. (2019) Active Tuberculosis Case Finding in IndiaThe Way Forward. Indian Journal of Tuberculosis, 66, 170-177.

https://doi.org/10.1016/j.ijtb.2018.05.014

[20] Vyas, A., Creswell, J., Codlin, A.J., Stevens, R., Rao, V.G., Kumar, B., et al. (2019) Community-Based Active Case-Finding to Reach the Most Vulnerable: Tuberculosis in Tribal Areas of India. The International Journal of Tuberculosis and Lung Disease, 23, 750-755. https://doi.org/10.5588/ijtld.18.0741

[21] Prathiksha, G., Daniel, B.D. and Natrajan, M. (2019) Active Case-Finding for Tuberculosis in India. The National Medical Journal of India, 32, 90-95. 